\title{
Effects of Hip Adductor Strengthening on Performance in Football Players
}

\author{
Arnaaz Rusi Doctor ${ }^{1 *}$ and Ali Irani ${ }^{2}$ \\ ${ }^{1}$ Physiotherapy Department, SVKM's NMIMS University, India \\ ${ }^{2}$ Head of Physiotherapy Department, SVKM's NMIMS University, India \\ *Corresponding Author: Arnaaz Rusi Doctor, Physiotherapy Department, SVKM's NMIMS University, India.
}

Received: September 23, 2019; Published: October 16, 2019

DOI: 10.31080/ASOR.2019.02.0114

\begin{abstract}
Introduction: Football is most widely spread and globally played sport today with kicking being one of the most fundamental ballbased football skill. The instep kick technique is used to achieve a high ball velocity while playing. The adductor longus is most commonly injured muscle during this sporting activity. The incidence has been reported to be 1.0 and1.1 per 1,000 playing hour. Prospective studies have shown an adductor strain incidence of $10 \%-18 \%$ of all injuries in football players. Hip adductors are one of the main muscles used while performing In-step and Front kicks, weakness of adductor group of muscle can make them more susceptible to injuries therefore it is important to strengthen the adductors, this study aims to show the effectiveness of hip adductor strengthening on performance in football players.

Methodology: As per inclusion and exclusion criteria, 32 subjects were included in this study, anthropometric measurements were recorded. They were assessed for Spot Instep kick distance, Spot Front kick distance and Kick velocity. Intervention program consisting of 8 Adductor strengthening exercises was incorporated for four weeks on the subjects. Post intervention evaluation was conducted with the same outcome measures. Result were recorded and analyzed using Paired T-test.

Conclusion: Hip adductor strengthening is effective in enhancing the performance of football players. There was a significant improvement in parameters of Instep kick distance, Front kick distance and Kick velocity.

Keywords: Adductor Strengthening; Instep Kick Distance; Front Kick Distance; Kick Velocity
\end{abstract}

\section{Abbreviations}

BMI: Body Mass Index; KD: Kick Distance; KV: Kick Velocity; PREISKD: Pre Instep Kick Distance; POSTISKD: Post Instep Kick Distance; PREFKD: Pre Front Kick Distance; POSTFKD: Post Front Kick Distance; PREKV: Pre Kick Velocity; POSTKV: Post Kick Velocity; EMG: Electromyography

\section{Introduction}

Football is most widely spread and globally played sport today. According to World Football report 2018, a survey was conducted across the world and football was voted by 736 million people across the surveyed markets as the most popular sport. In India, cricket dominates but football has been gaining ground. A big engine of growth in recent years has been the Indian Super League, a nationwide professional league launched in 2013 [1].

Statistical survey was conducted by Cint on the kind of sports respondents in India, 2018. During the survey it was found that 4.69 percent of Indian respondents play football regularly. Earlier football was only played for recreation but now it has been evolved as a growing profession in India.

Football is a high impact sport with kicking being one of the most fundamental ball-based football skill. It is a complex motor 
movement consisting of six important stages: Approach angle, plant foot forces, swing limb loading, flexion at the hip and extension at the knee, foot contact with the ball, and follow-through. The kicking procedure may also be divided into five essential aspects: Approach, support leg, kicking leg, foot to ball interaction, and ball flight [2]. The instep kick technique is used to achieve high ball velocity and long trajectory. Kicking is a crucial moment in every match since its number and efficiency influence the final score and it is necessary to realize that speed and accuracy of kick are influenced by various factors. The football kick speed is mainly affected by the angle of running towards the ball. Kicking accuracy is influenced by many factors ranging from mistakes in a player's approach to the kick, position of the supporting leg during the kick, movement of the kicking leg - its swing and placing the foot on the ball. Ball speed depends on the velocity of the foot upon impact as well as the quality of ball - foot impact. The supporting leg is considered to be responsible for whole body stabilization therefore it has a positive effect on kicking performance as well [3].

The instep kick constitutes a basic element of football game and it depends on the strength of adductor group of muscles and also the angle of approach to the ball. The in-step kick is widely used as main offensive, technical action during the game and also to produce faster ball velocity than other kicking techniques, thereby giving the goal keeper less time to react, thus expanding one's chance of scoring [4].

The primary function of adductor muscle group is adduction of the thigh in open chain motions and stabilization of the lower extremity and pelvis in closed chain motion. Hip abduction involves the gluteus medius and gluteus minimus, while the longus, brevis, magnus, and gracilis perform hip adduction [5]. The adductor longus being the most commonly injured muscle during sporting activity [4].

Adductor-related groin pain is defined by pain on palpation and isometric contraction of the adductors and is the most common clinical symptom seen in elite and sub-elite football players. Hip adductor and hip flexor weakness have been suspected of being associated with this condition. In athletes with nonspecific groin pain, it seems that bilateral isometric hip adductor strength is decreased by $20 \%$ to $25 \%$ compared with asymptomatic controls [6].
In football adductor muscle injury is most commonly seen, the incidence being reported to be 1.0 and1.1 per 1,000 playing hour and prospective studies showing an adductor strain incidence of $10 \%-18 \%$ of all injuries in football players. This means that a male professional football team will suffer an average of seven groin injuries per season, with greater than $50 \%$ of them being unavailable for selection for at least one week [4].

Hip adductors are one of the main muscles used while performing on In-step and Front kicks. Weakness of adductor group of muscle can make them more susceptible to injuries therefore it is important to strengthen the adductors, this study aims to show the effectiveness of hip adductor strengthening on performance in football players as importance of adductor group muscles in football players is under-explored.

\section{Materials and Methods}

This is an interventional study done on 32 healthy male football players of adult age group (above 18 years) with atleast one year of professional coaching in Mumbai by method of convenience sampling. The football players with recent lower limb injuries (6 months) like soft tissue injury, fractures, sprains, strains were excluded.

The study procedure for this study was as follows. Ethical approval was taken to conduct this study at Nanavati Super Speciality Hospital, India. 42 subjects were selected as per the inclusion and exclusion criteria, consent was taken to participate in this study after which anthropometric measurements such as height, weight and BMI were noted down.

After that subjects were assessed for the outcome measures: Spot kick distance and kick velocity. For assessing kick distance the players were asked to kick in 2 ways, In step kick and Front kick. The scoring was done by measuring the distance from the point of kick till the point of first contact using a measuring tape.

Bushnell velocity speed gun radar was used to access the kick velocity. The radar was placed half a meter behind the goal post to measure the velocity of kick. Speed was recorded in Kph (Kilometers per hour). 
An intervention program consisting of 4 Adductor strengthening exercises was incorporated. The exercises were as follows: Adductor isometrics, Side lying Adductor, Adductor strengthening with resistance bands in standing and Sumo squats with resistance band. As per the American college of sports medicine guidelines, 3 sets of 10 repetitions, four times a week for 2 weeks was done.

The first 2 weeks exercises were performed as follows: (1) Adductor isometrics: Subject was in supine on the mat with hip and knee at $90^{\circ}$ Flexion with legs in air. A ball was placed between the knees and subject had to squeeze the ball and hold for 5seconds. (2) Sidelying adductor: Subject was in sidelying position. The upper leg's knee was flexed and put over the lower leg with feet touching the ground. Subject was asked to lift lower leg without bending knee and hold for 5seconds. (3) Adductor strengthening with resistance band in standing: The band was tied at the ankle of the subject. Subject had to take his leg inside and cross over the other leg and hold for 5seconds. (4) Sumo squats with resistance band: A Resistance band was tied at the ankle and the subject was asked to perform sumo squats with feet pointed outwards.

After which 4 more exercises which included Sidelying Adductor with chair, $\mathrm{V}$ sitting long arm adductor, Adductor lunges and Adductor strengthening with stepper was added, for the next 2 weeks. The exercises were performed as follow: (5) Sidelying with chair: Subject was in side lying position with upper leg kept on the chair. The subject had to lift his lower leg straight with knees straight and hold for 5seconds. (6)V sitting long arm adductor: Subject was in V-sitting position with a ball squeezed between feet. Subject had to perform knee bending and hold for 5 seconds. (7) Adductor lunges: The subject had to stand on one leg and drag the other leg at the side with feet out and perform a lunge and hold for 5seconds. (8) Adductor strengthening with stepper: Subject was made to stand with his non-dominant leg on the stepper and with the dominant leg on the ground drag a resistance ball inside.

In total 8 Adductor strengthening exercises were performed for the next 2 weeks, four times a week. Post eight weeks due to loss of follow up 32 subjects were evaluated with the same outcome measures and results were recorded.

For statistical analysis of the data recorded, statistical package of social sciences (SPSS) version 23 was used setting the level of significance at 0.05 . Mean and standard deviation of Instep kick distance, Front kick distance and Kick velocity was calculated. Paired $t$ test was used for analysis of all outcome measures.

\section{Results and Discussion}

The aim of this study was to show effectiveness of Hip Adductor strengthening on performance in football players. The main outcome of this study was to analyse the effectiveness of hip adductor strengthening on Instep kick distance, Front kick distance and Kick velocity in enhancing their performance.

\section{Demographic Data}

This study was conducted on 32 football players. As seen in figure 1, maximum players in this study were between 21 to 24 years of age, $16 \%$ were below 20 years of age, $13 \%$ were between 25 to 30 years of age and only $9 \%$ were above 30 years of age.

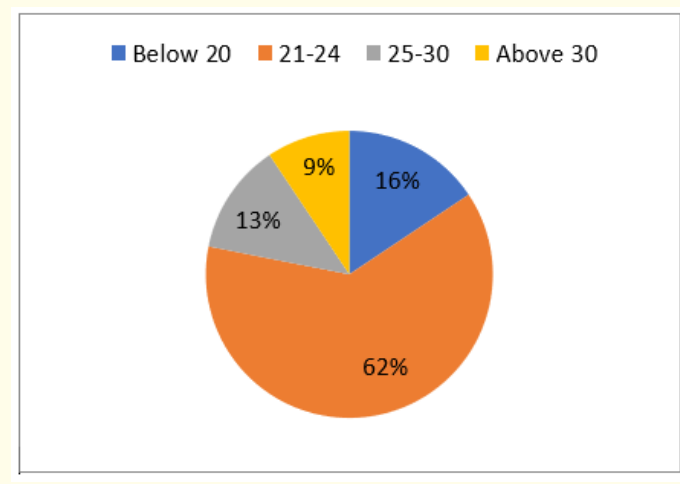

Figure 1: Age Distribution in the study.

An average male player peaks between ages 25 and 27 [7], the player will perform at his maximum level during this period. As football is a prototypical team game therefore the extent to which a player expresses his ability still largely depends on the players surrounding him.

BMI

Figure 2, table 1 depicts the Body Mass Index distribution in our study, where maximum players were within the normal BMI range according to WHO guidelines. $12 \%$ were underweight, $18 \%$ were over-weight whereas $3 \%$ were in obese category.

Elite football is a complex sport requiring high intensity bouts that are performed above the lactate threshold where strength, agility, speed and endurance are important components therefore 
performance of the players depends on factors such as physical fitness, psychological factors, player's technique and team tactics. It is a game which requires very fast body movement which includes short and long sprints, explosive reactions (jump) and quick changes of direction. High-speed actions are known to impact performance and require maximal speed, acceleration or agility. There is a significant relationship between BMI with speed and BMI with agility therefore it is important for football players to be within the normal BMI range to perform well [8].

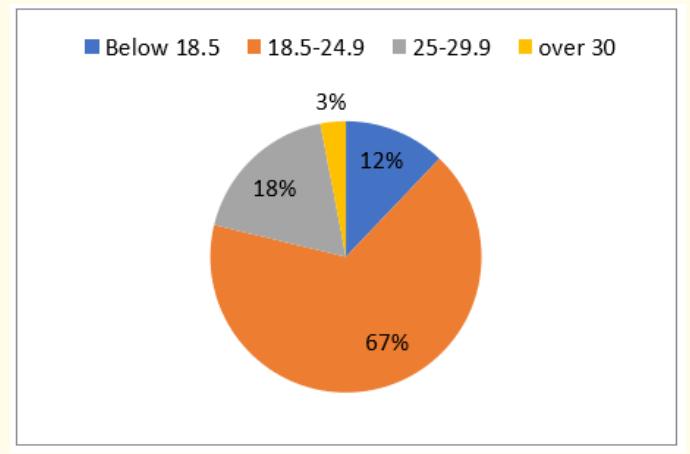

Figure 2: BMI Distribution in the study.

\begin{tabular}{|l|c|c|}
\hline Below 18.5 & Underweight & $\mathbf{1 2 \%}$ \\
\hline $18.5-24.9$ & Normal & $67 \%$ \\
\hline $25-29.9$ & Overweight & $18 \%$ \\
\hline Above 30 & Obese & $3 \%$ \\
\hline
\end{tabular}

Table 1: BMI distribution according to WHO.

\section{Kick distance}

Table 2 and figure 3 indicate from pre mean $41.650 \pm 1.05 \mathrm{~m}$ to post mean $43.466 \pm 1.07 \mathrm{~m}$ after the adductor strengthening intervention protocol, there was improvement in the Instep kick distance.

\begin{tabular}{|l|c|c|}
\hline Preiskd & Postiskd & P Value \\
\hline $41.650 \pm 1.05 \mathrm{~m}$ & $43.466 \pm 1.07 \mathrm{~m}$ & 0.00 \\
\hline
\end{tabular}

Table 2: Instep Kick Distance.

Referring to table 3 and figure 4 from pre mean $41.688 \pm 6.27 \mathrm{~m}$ to post mean $45.303 \pm 5.53$ after the adductor strengthening intervention protocol, there was improvement seen in the Front kick distance.

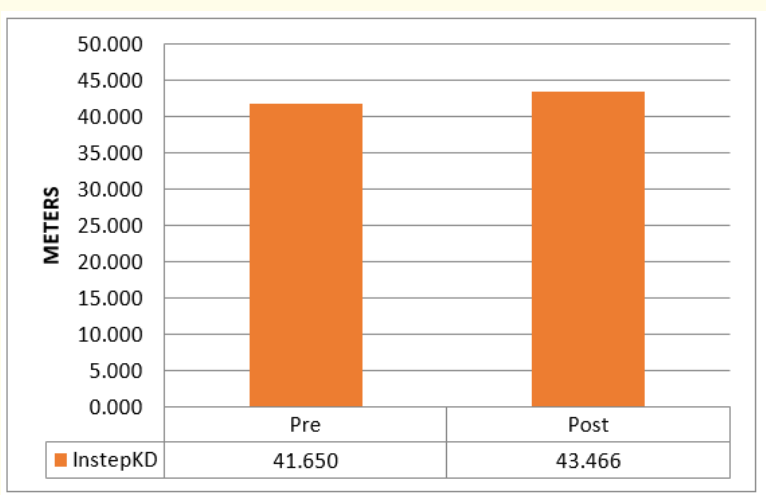

Figure 3: Instep Kick Distance.

\begin{tabular}{|l|c|c|}
\hline Prefkd & Postfkd & P Value \\
\hline $41.688 \pm 6.27 \mathrm{~m}$ & $45.303 \pm 5.53 \mathrm{~m}$ & 0.00 \\
\hline
\end{tabular}

Table 3: Front Kick Distance.

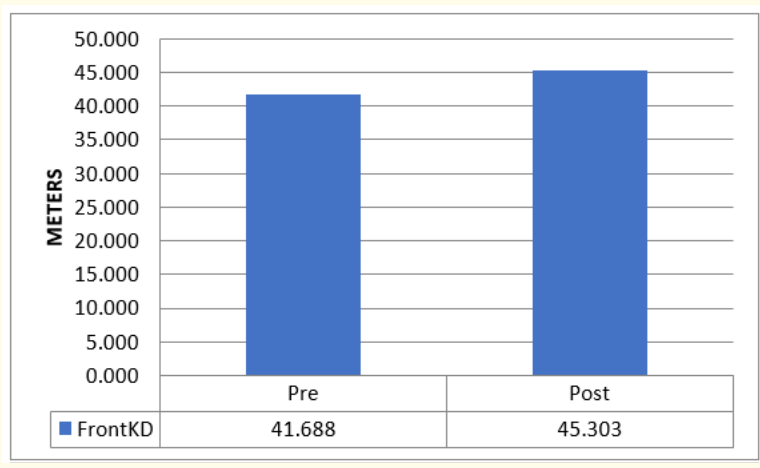

Figure 4: Front Kick Distance.

The instep kick to striking a ball is the foundation of shooting with power and precision. It is considered the most important kicking skill due to the loading and unloading of physical movements within a kinetic chain that transfer high amounts of power into the kick, which in turn forces the player to drive the ball forward. This kick is highly regarded as it forms the basis for learning other kicking techniques such as the chip, clearances, volleys, long passes, goal and corner kicks.

EMG analysis of hip adductor muscles in football instep kick has shown increase activity in the AM muscle of the kicking leg while 
in the supporting leg both AM and AL muscles was significantly increased during instep kick. This result suggests that hip adductor muscles effectively contribute to either the kicking or supporting leg to enhance the action of football kicks [9].

Groin injury is the most common injury seen in football players, representing $9 \%-16 \%$ of all injuries. They often cause problems such as high rates of recurrence, prolonged absence from the game and training sessions, uncertain prognosis and long-term symptoms. Previous reports have also indicated that $69 \%$ of groin injuries in football players are adductor-related [10]. Adductor longus using three-dimensional joint positions and muscle activation was obtained which showed the maximum rate of stretch of the adductor longus and maximum hip extension was at near $40 \%$ of swing phase. There was activation of the adductor longus seen between $10 \%$ and $50 \%$ of the swing phase. Adductor longus maximum length occurred at $65 \%$ of the swing phase therefore Adductor longus is the most common muscle to get strained during maximal effort football kick [11]. Adductor Strengthening has shown to substantially reduce the self-reported prevalence and risk of groin problems in male football players where the risk of reporting groin problems was $41 \%$ lower in the intervention group than the control group [12].

The adductor isometric exercise involves the static contraction of a muscle without any visible movement in the angle of the joint. In this exercise the length of the muscle and the angle of the joint do not change, though contraction strength may be varied. The side-lying hip adduction and adductor ball squeeze shows high activation level of the adductor longus muscle [13]. Also it is seen that hip-adduction strength training performed with elastic bands as external load increases maximal eccentric hip-adduction strength substantially in healthy sub-elite football players [14]. EMG study have also shown significant activation in Adductor Longus muscle during Copenhagen exercise (Adduction exercise with chair), hence this exercise plays an important role in strengthening the adductor group [15].

Since all these exercises cause activation of adductor muscles therefore they are incorporated in this protocol to strengthen them. This study depicts that hip adductor strengthening has shown statistical improvement in Instep and Front Kick Distance.

\section{Kick velocity}

Table 4 and figure 5 depicts pre mean $80.03 \pm 8.52 \mathrm{kph}$ to post mean $85.06 \pm 7.24 \mathrm{kph}$ after adductor strengthening intervention protocol, there was improvement in the Kick Velocity.

\begin{tabular}{|l|c|c|}
\hline Prekv & Postkv & P Value \\
\hline $80.03 \pm 8.52 \mathrm{kph}$ & $85.06 \pm 7.24 \mathrm{kph}$ & 0.00 \\
\hline
\end{tabular}

Table 4: Kick Velocity.

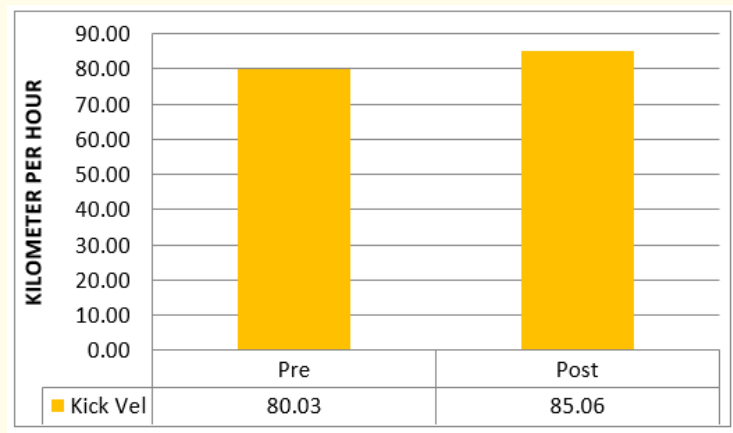

Figure 5: Kick Velocity.

The hip adduction exercises with a resistive band increases trunk stability. Strengthening the adductor group of muscles also in turn strengthen the core. The co-activation of hip adductors, pelvic floor and abdominals is necessary for occurrence of intra-abdominal pressure. Therefore an optimal hip adduction exercise strengthens the hip adductor muscles and enhances core stability [16]. The sitting $\mathrm{V}$ long arm adductor exercise also requires co-activation of the core muscle with adductor group muscle while performing knee flexion and extension. In the adductor strengthening with stepper exercise, the muscles of stationary leg are working to maintain balance as well as there is constant core activation to maintain trunk stability on the stepper which provides additional resistance while the other leg's adductor muscles are working during the exercise.

In the anterior oblique system of the body, the thigh adductors are coupled with abdominals through fascia therefore trunk movement first lengthens the muscles and energy is stored. This energy is released during concentric contraction of adductor muscles allowing greater velocity. A high amount of eccentric work is done by hip adductors during loading phase of the kick. The stored en- 
ergy is subsequently released during the swing phase to generate greater kicking velocities [17].

Therefore adductor strengthening has shown improvement in the kick velocity thereby enhancing the performance of football players.

\section{Conclusion}

The conclusion of this study is that Hip adductor strengthening is effective in enhancing the performance of football players. There was a significant improvement in parameters of Instep kick distance, Front kick distance and Kick velocity therefore adductor strengthening exercises should be incorporated in the regular football training.

\section{Acknowledgements}

I am grateful to the Almighty God for establishing me to complete this research. I express my heart full indebtedness and owe a deep sense of gratitude to Dr. Poonam Parulekar, Dr. Mansi Bhartiya, Dr. Sabah Thaver and Dr. Khadijeh Mennai for their constant support and guidance. I am very much thankful to my parents (Rusi and Rashna Doctor) who guided and encouraged me in each and every step. My cordial gratitude to my friends Zoraius Kamdin and Vahista Sui, their support and encouragement was a great help throughout the course of this research work. Needless to mention, I would also like to thank all my study subjects for their immense cooperation. I will be failing in duty if I do not acknowledge with grateful thanks to the authors of the references and other literatures referred to in this study.

\section{Conflict of Interest}

None.

\section{Bibliography}

1. Nielson World Football Report (2018).

2. Cerrah Ali Onur., et al. "The effect of kick type on the relationship between kicking leg muscle activation and ball velocity". Montenegrin Journal of Sports Science and Medicine 7.1 (2018): 39-44

3. Kellis, Eleftherios, and Athanasios Katis. "Biomechanical characteristics and determinants of instep soccer kick". Journal of Sports Science and Medicine 6.2 (2007): 154.

4. Griffin Victoria Charlotte., et al. "A comparison of hip adduction to abduction strength ratios, in the dominant and nondominant limb, of elite academy football players". Diss. Cardiff University (2011).

5. Renström PAFH and LPeterson. "Groin injuries in athletes". British Journal of Sports Medicine 14.1 (1980): 30.
6. Thorborg, Kristian, et al. "Eccentric and isometric hip adduction strength in male soccer players with and without adductor-related groin pain: an assessor-blinded comparison". Orthopaedic Journal of Sports Medicine 2.2 (2014): 2325967114521778.

7. Dendir Seife. "When do soccer players peak? A note”. Journal of Sports Analytics 2.2 (2016): 89-105.

8. Dhapola Mahesh Singh and Bharat Verma. "Relationship of body mass index with agility and speed of university players." (2017).

9. Watanabe Kohei., et al. "Electromyographic analysis of hip adductor muscles in soccer instep and side-foot kicking". Sports Biomechanics (2018): 1-12.

10. Yousefzadeh Abbas., et al. "The Effect of Therapeutic Exercise on Long-Standing Adductor-Related Groin Pain in Athletes: Modified Hölmich Protocol". Rehabilitation Research and Practice (2018).

11. Charnock Brian L., et al. "Adductor longus mechanics during the maximal effort soccer kick". Sports Biomechanics 8.3 (2009): 223-234.

12. Harøy Joar., et al. "The Adductor Strengthening Programme prevents groin problems among male football players: a cluster-randomised controlled trial." British Journal of Sports Medicine 53.3 (2019): 150-157.

13. Delmore Robert J., et al. "Adductor longus activation during common hip exercises". Journal of Sport Rehabilitation 23.2 (2014): 79-87.

14. Jensen Jesper., et al. "Eccentric strengthening effect of hipadductor training with elastic bands in soccer players: a randomised controlled trial". British Journal of Sports Medicine 48.4 (2014): 332-338.

15. Serner Andreas., et al. "EMG evaluation of hip adduction exercises for soccer players: implications for exercise selection in prevention and treatment of groin injuries". British Journal of Sports Medicine 48.14 (2014): 1108-1114.

16. Kim Min-hee and Won-gyu Yoo. "Optimal and Maximal Loads during Hip Adduction Exercise by Asymptomatic People". Journal of Physical Therapy Science 26.5 (2014): 777-778.

17. Bram Swinnen. Stregth Training for Soccer Book 57.

\section{Volume 2 Issue 11 November 2019 (C) All rights are reserved by Arnaaz Rusi Doctor and Ali Irani.}

